\title{
Imbibition in Mesoporous Silica: Rheological Concepts and Experiments on Water and a Liquid Crystal
}

\author{
Simon Gruener ${ }^{1,2}$ and Patrick Huber ${ }^{1}$ \\ ${ }^{1}$ Experimental Physics, Saarland University, D-66041 Saarbrücken, Germany \\ 2 Department of Mechanical and Aerospace Engineering, Princeton University, \\ Princeton, NJ 08544, USA \\ E-mail: sgruner@princeton.edu, p.huber@physik.uni-saarland.de
}

\begin{abstract}
Along with some fundamental concepts regarding imbibition of liquids in porous hosts we present an experimental, gravimetric study on the capillarity-driven invasion dynamics of water and of the rod-like liquid crystal octyloxycyanobiphenyl (8OCB) in networks of pores a few nanometers across in monolithic silica glass (Vycor). We observe, in agreement with theoretical predictions, square-root of time invasion dynamics and a sticky velocity boundary condition for both liquids investigated. Temperature-dependent spontaneous imbibition experiments on $8 \mathrm{OCB}$ reveal the existence of a paranematic phase due to the molecular alignment induced by the pore walls even at temperatures well beyond the clearing point. The ever present velocity gradient in the pores is likely to further enhance this ordering phenomenon and prevent any layering in molecular stacks, eventually resulting in a suppression of the smectic in favor of the nematic phase.
\end{abstract}

PACS numbers: 47.55.nb, 47.61.-k, 87.19.rh, 47.57.Lj, 47.56.+r 


\section{Introduction}

Liquid flow propelled by capillary forces is one of the most important transport mechanisms in porous environments. It is governed by a fascinating interplay of interfacial, viscous drag as well as gravitational forces which liquids encounter upon invasion into geometries with often complex topologies, such as capillary networks of trees [1-3] or interconnected fractures in soils and ice. There has been significant progress with regard to a quantitative understanding of this phenomenon, most prominently with regard to the transport through rocks and soils, starting with the seminal work of the French engineer Henry Darcy [4,5. Almost all experimental and theoretical studies, published to date, deal however with porous structures where the characteristic length scales, meaning the typical pore diameters, are on the macroscale, that is much larger than the typical size of the basic building blocks (molecules) of the liquids 6.

After an introduction of the theoretical and experimental fundamentals, exemplified for the case of water imbibition in mesopores, we present a temperature-dependent imbibition study on the liquid crystal octyloxycyanobiphenyl (8OCB). The goal is to scrutinize which principles regarding its rheology, known from the bulk state, can be safely transferred to the transport across nano- or mesoscopic geometries. Finally, insights into the phase transition behavior of the spatially nano-confined, rod-like liquid crystal will be gained.

Given the emerging interest in micro- and nanofluidic applications [7] such a study on the non-equilibrium behavior of liquids in extreme spatial confinement is not only of fundamental interest, but also of rather practical importance [8 10]. Moreover, the increasing use of mesoporous matrices as hard templates [11 for the preparation of well-defined nanoscopic, soft-matter structures, such as nanotubes and nanorods [12], necessitates a profound understanding of this phenomenology.

\section{Fundamentals of liquid imbibition in mesoporous solids}

\subsection{Liquid flow in isotropic pore networks: Darcy's law}

In order to introduce the fundamentals of liquid flow in mesoporous media, we start with a much simpler phenomenology: The flow of a liquid through a single straight pipe. According to the law of Hagen-Poiseuille the volume flow rate $\dot{V}_{\mathrm{HP}}$ for a given pressure difference $\Delta p$ applied along the cylindrical duct with radius $r$ and length $\ell$ is determined by

$$
\dot{V}_{\mathrm{HP}}=\frac{\pi r^{4}}{8 \eta \ell} \Delta p
$$

Here $\eta$ denotes the dynamic viscosity of the liquid. In the following we will present a simple concept that allows one to extend the applicability of Eq. (1) to the flow through a complex pore network [1]. 
Porous Vycor glass (code 7930) provided by Corning Incorporated was used over the course of this study. It is produced through metastable phase separation in an alkali-borosilicate system followed by an extraction of the alkali-rich phase. The final glass consists of a sponge-like network of tortuous and interconnected pores with mean pore radii on the nanometer scale embedded in a matrix mostly consisting of $\mathrm{SiO}_{2}[13]$.

Such an isotropic pore network can be characterized by a set of three quantities. The pore radius $r_{0}$ and the volume porosity $\phi_{0}$ are probably the most intuitive ones among them. Two different batches of Vycor glass were applied in this study that differed in $r_{0}$ whereas they coincided in $\phi_{0}=0.32 \pm 0.01$. We will refer to them as V5 $\left[r_{0}=(3.4 \pm 0.1) \mathrm{nm}\right]$ and V10 $\left[r_{0}=(4.9 \pm 0.1) \mathrm{nm}\right]$ from now on 14$]$.

These matrix properties were accurately ascertained employing nitrogen sorption isotherms conducted at $77 \mathrm{~K}$ and through a subsequent analysis within a mean field model for capillary condensation in nano- and mesopores [15]. We obtained pore size distributions $P(r)$ with standard deviations of approximately $0.1 r_{0}$ (with the peak or most probable value of the distribution $r_{0}$ ). In order to assess the impact of this uncertainty in $r$ we turn to the quantity measured in our experiments that is the mass increase of the porous matrix due to the uptake of a liquid - see section 3 for details. Here each pore gives a signal that is proportional to its volume $\propto r^{2}$ and consequently the macroscopic signal, which is averaged over the complete sample, should be identically equal to the result for an imaginary porous host with the single pore radius

$$
r^{\prime} \equiv \sqrt{\frac{\int r^{2} P(r) \mathrm{d} r}{\int P(r) \mathrm{d} r}} .
$$

Since we could not find any difference between $r_{0}$ and $r^{\prime}$ exceeding their error margins we conclude that the distributions can be considered to be narrow and negligible in these specific measurements. Hence we proceed taking into consideration only the most probable pore radii $r_{0}$ stated above.

To account for the isotropy of the network the so-called tortuosity $\tau$ must be introduced along with the transformation $\dot{V} \rightarrow \tau^{-1} \dot{V}$. Pores totally aligned in flow direction yield $\tau=1$, whereas isotropically distributed pores result in $\tau=3$ since for randomly oriented channels only every third pore is subjected to the pressure gradient and hence contributes to the flow. To date many different methods have been applied in order to extract the tortuosity of porous Vycor glass 16 18. They consistently yield $\tau \approx 3.6$ which, interestingly, deviates from $\tau=3$. This is because the pores are not straight but rather meandering. A value $\tau>3$ corrects the pore length for the larger flow path.

Finally one is able to derive an expression that describes the flow of a liquid through a porous network. For a given porous matrix with cross-sectional area $A$ and thickness $d$ (along which the pressure drop $\Delta p$ is applied) the volume flow rate $\dot{V}_{\mathrm{D}}$ is determined by Darcy's law 19

$$
\dot{V}_{\mathrm{D}}=A \frac{\phi_{0} r_{0}^{2}}{8 \tau \eta d} \Delta p .
$$




\subsection{Velocity boundary conditions}

The law of Hagen-Poiseuille implies the no-slip boundary condition. This means that the velocity of the fluid layers directly adjacent to the restricting walls equal the velocity of the walls themselves. Nowadays it is indisputable that this assumption does not hold unreservedly. Already 60 years ago Peter Debye and Robert Cleland introduced both slipping and sticking fluid layers at the pore walls in order to interpret a seminal experiment on liquid flow across porous Vycor [19].

Up to date many factors have been found that seem to influence the boundary conditions $20-22]$. The least controversially discussed amongst them is the fluid-wall interaction $[23[34]$. Shear rates beyond a critical value are supposed to induce slip, too 35 38. In contrast, the influence of surface roughness is rather debatable 24, 39 41. Furthermore, dissolved gases [20, 42], the shape of the fluid molecules [28] or the add-on of surfactants 43 might influence the boundary conditions as well.

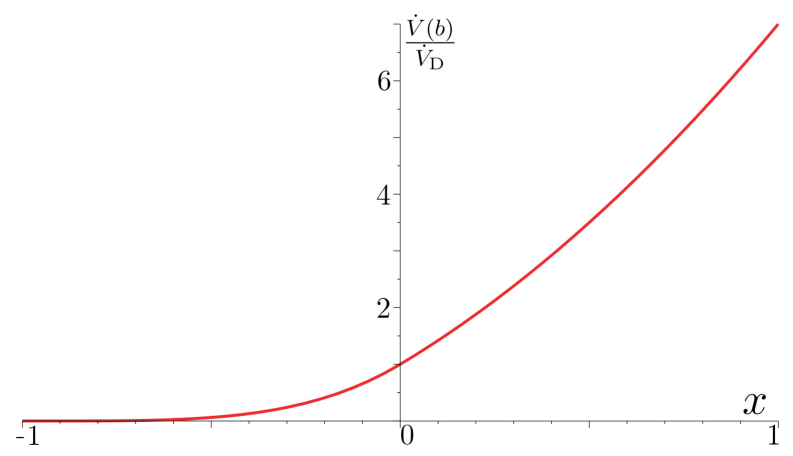

Figure 1: Volume flow rate $\dot{V}(b)$ normalized with Darcy's flow rate $\dot{V}_{\mathrm{D}}$ Eq. (3) as a function of the slip length $b$ in units of the pore radius $r_{0}: x \equiv \frac{b}{r_{0}}$. Note that the flow rate vanishes for $b \rightarrow-r_{0} \Leftrightarrow x \rightarrow-1$.

The degree of slip can be quantified by the slip length $b$ with $r_{\mathrm{h}} \equiv r_{0}+b$ the hydrodynamic pore radius, which measures the distance from the pore center to the radius where the streaming velocity reaches zero. Thus, a sticking layer boundary condition corresponds to $b<0$. The volume flow rate $\dot{V}(b)$ can be calculated from the parabolic velocity profile

$$
v_{\mathrm{z}}(r)=\frac{\Delta p}{4 \eta \ell}\left[\left(r_{0}+b\right)^{2}-r^{2}\right]
$$

through an integration over the channel's cross-sectional area $\pi r_{0}^{2}$ if $b>0$. For $b<0$ however the immobile layers have to be excluded. Then $\dot{V}(b)$ in units of $\dot{V}_{\mathrm{D}}$ is given by

$$
\frac{\dot{V}(b)}{\dot{V}_{\mathrm{D}}}= \begin{cases}(x+1)^{4} & \text { if } \quad b<0 \\ 1+4 x+2 x^{2} & \text { if } \quad b>0\end{cases}
$$

in terms of normalized slip lengths $x \equiv \frac{b}{r_{0}}$. In Fig. 1 this quantity is plottet for $-r_{0}<b<r_{0}$. Because of the obvious bijectivity of $\dot{V}(b)$ any measured flow rate $\dot{V}$ can unambiguously be related to a unique slip length $b$. Part of the evaluation of the present investigation is based upon this principle. 


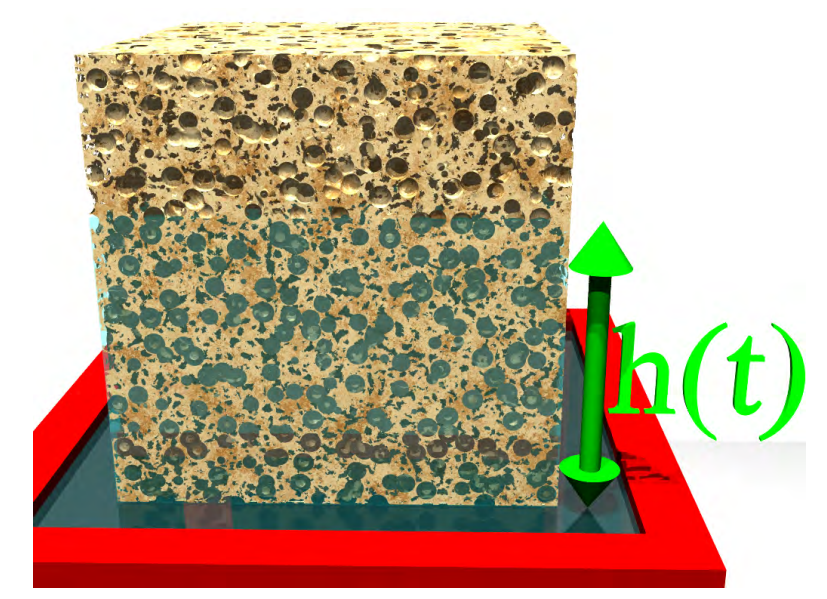

Figure 2: Raytracing illustration of a Vycor sample during a capillary rise experiment filled up to the level $h(t)$.

\subsection{Dynamics of spontaneous imbibition}

From the physisist's point of view spontaneous imbibition is an impressive example for interfacial physics. The driving force behind the capillary rise process is the Laplace pressure $p_{\mathrm{L}}$ acting on the curved meniscus of a liquid in a pore or porous structure. It is specified by

$$
p_{\mathrm{L}}=\frac{2 \sigma \cos \theta_{0}}{r_{0}}
$$

with $\sigma$ the surface tension of the liquid. This implies that spontaneous imbibition can only occur for a wetting or partially wetting liquid.

As the liquid rises beyond its bulk reservoir to a certain level $h$ the hydrostatic pressure $p_{\mathrm{h}}=\rho g h$ acting on the liquid column increases. The final state is derived from a balance between $p_{\mathrm{L}}$ and $p_{\mathrm{h}}$ [4] resulting in the maximum rise level given by Jurin's law. However, because of the tiny pore diameters considered in our study typical Laplace pressures are on the order of a few 100 bar and hence much higher than typical hydrostatic pressures. The gravitational force may therefore be neglected.

In order to gain information on the dynamics of the imbibition process in a porous network we can now resort to Darcy's law. Considering Fig. 2 one easily concludes that the sample height $d$ appearing in Eq. (3) has to be replaced by the time-dependent rise level $h(t)$ since only the filled parts of the sample contribute to the flow dynamics. Moreover, at any given time $t$ both the imbibed fluid volume $V(t)$ and its mass $m(t)$ are closely related to $h(t)$ (utilizing the fluid's density $\rho$ ) via

$$
V(t)=\frac{m(t)}{\rho}=\phi_{0} A h(t) .
$$

Eventually this leads to the simple differential equation

$$
\dot{h}(t) h(t)=\frac{r_{0}^{2}}{8 \tau \eta} \Delta p
$$


solved through

$$
h(t)=\sqrt{\frac{r_{0}^{2}}{4 \tau \eta} \Delta p} \sqrt{t}
$$

the Lucas-Washburn (LW) law 45,46]. Substituting the Laplace pressure for $\Delta p$ and utilizing Eq. (7) finally yields

$$
m(t)=\underbrace{\rho A \phi_{0} \sqrt{\frac{r_{0} \sigma}{2 \tau \eta}}}_{C} \sqrt{t},
$$

the mass increase $m(t)$ of the sample due to the liquid uptake as a function of the timeł. It is noteworthy that molecular dynamics simulations [47,48 corroborate on a microscopic scale the validity of this model for imbibition of molecular fluids as derived from macroscopic, rather phenomenological considerations even down to mesoand nanopores.

The imbibition coefficient $C$ is a simple measure of the invasion dynamics. Please note that the above equation explicitly obeys the no-slip boundary condition. Hence, any deviation of a measured imbibition coefficient from the one predicted according to Eq. (10) may directly be interpreted in terms of a non-zero slip length $b$ utilizing Eq. (5).

\section{Experimental Part}

Imbibition dynamics are studied via a recording of the samples' mass increase $m(t)$. The setup is depicted in Fig. 3. For a time-dependent measurement of the force acting on and, hence, of the mass increase of the porous Vycor block the sample is installed on standard laboratory scales applying a special mounting. In order to perform measurements beyond room temperature a cell was constructed that allows for a simultaneous thermostatting of the sample itself and the liquid reservoir beneath.

Upon start of the experiment the cell is moved upward until the sample touches the liquid surface. The liquid immediately starts invading the porous host what becomes noticeable by the gradual increase of the sample's mass (see Fig. 4). According to the previously introduced model of imbibition and Eq. (10) this behavior can be very well described by a $\sqrt{t}$-fit (see solid line in Fig. 4), which yields the imbibition coefficient $C$. Eventually a level of saturation is entered, which signals that the sample is completely filled.

\subsection{Data interpretation using the example of mesopore-confined water}

Experiments on the capillary rise of water in V5 and V10 were conducted at three different temperatures $\mathrm{T}\left(25^{\circ} \mathrm{C}, 40^{\circ} \mathrm{C}\right.$, and $\left.60^{\circ} \mathrm{C}\right)$. The measured flow rates showed

$\ddagger$ Since all liquids considered in our study totally wet the high-energy silica surface of the pore walls (as confirmed by contact angle measurements in the group of Karin Jacobs at Saarland University, Saarbruecken, Germany) the contact angle is already eliminated because of $\cos \left(0^{\circ}\right)=1$. 


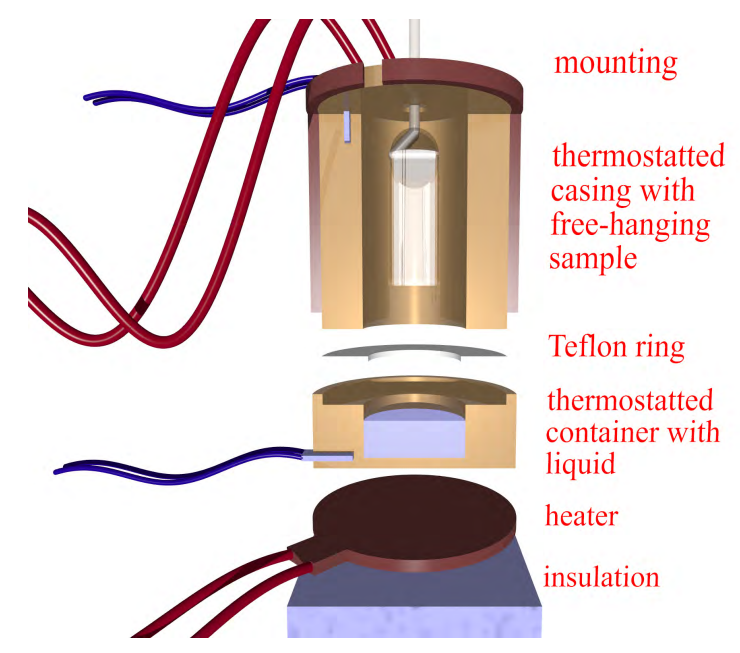

Figure 3: Exploded view of the imbibition setup (raytracing illustration). The top of the Vycor sample is glued to a wire and hangs freely into the cell. The cell itself consists of a casing and a container. Both are build out of copper and can separately be thermostatted. The cell itself can be moved in vertical direction.

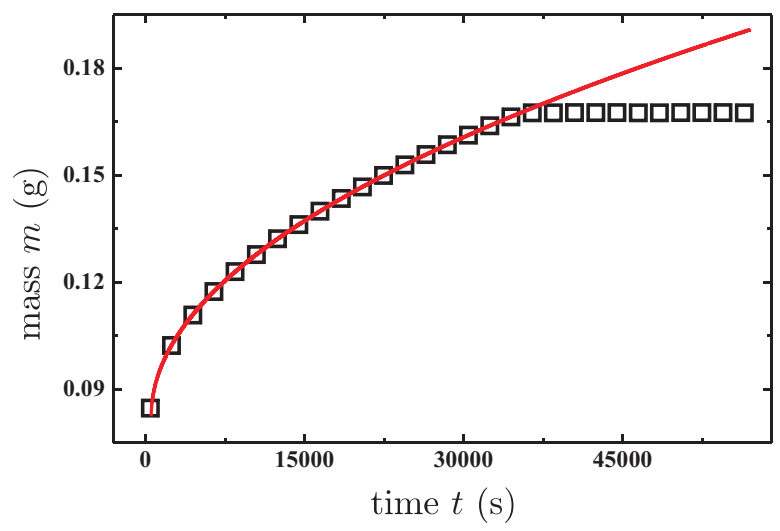

Figure 4: Measurement of the mass increase of a porous Vycor block upon the liquid invasion during an imbibition experiment. The solid line is a $\sqrt{t}$-fit according to Eq. (10). The data density is reduced by a factor of 2000 .

significant deviations from the expected value according to Eq. (10). Utilizing Eq. (5) this discrepancy could be transferred into a non-zero slip length. Consistently for both sample batches a negative slip length of about $5 \AA$ was found. Considering the diameter of a water molecule of approximately $2.5 \AA$ this result can be interpreted as follows: two layers of water directly adjacent to the pore walls are immobile meaning that they are pinned and do not take part in the flow. The residual inner compartment of the liquid however obeys classical hydrodynamics in accordance with former findings on the conserved fluidity [49,51] and capillarity [52,53] of confined water.

The idea of the compartmentation of mesopore-confined water is corroborated by recent molecular dynamics studies on the glassy structure of water boundary layers 
in Vycor and the expected existence of sticky boundary layers in Hagen-Poiseuille nanochannel flows for strong fluid-wall interactions [54 59. By means of X-ray diffraction distortions of the hydrogen-bonded network of water near silica surfaces were found [60], which might be responsible for the markedly altered liquid properties. Tip-surface measurements document a sudden increase in the viscosity by orders of magnitude in $0.5 \mathrm{~nm}$ proximity to hydrophilic glass surfaces 61 63. It also extends former experimental results with respect to the validity of the no-slip boundary condition for water/silica interfaces, in which this condition was proven down to at least $10 \mathrm{~nm}$ from the surface [64] whereas slip-flow for water is only expected at hydrophobic surfaces 64, 65]. For more detailed information we would like to refer the interested reader to two publications reporting on capillary rise dynamics of water in mesoporous silica 66, 67].

It is interesting to note that such a compartmentisation of the pore fluid with respect to its dynamics has also been inferred for the linear n-alkanes confined in mesopores, both from imbibition experiments [68] and from neutron-spin echo experiments on the self-diffusion dynamics [69].

\section{Rheology and phase transition behavior of the liquid crystal 8OCB in mesopore-confinement}

Usually phase transitions are accompanied by unique variations of the fluid properties. Since the capillary rise dynamics, and in particular the imbibition coefficient $C$, sensitively depend on these quantities one can easily detect such characteristic deviations by performing measurements at different temperatures in the $T$-range of interest. It is convenient to normalize the extracted prefactors $C(T)$ with the value $C_{\mathrm{n}} \equiv C\left(T_{\mathrm{n}}\right)$ at an arbitrarily chosen temperature $T_{\mathrm{n}}$. This procedure eliminates all quantities that do not depend on the temperature. Particularly, the geometry $(A)$ as well as the internal topology of the substrate $\left(r_{0}, \phi_{0}\right.$, and $\left.\tau\right)$ do not play a role any longer. Only the fluid properties $\sigma(T), \eta(T)$, and $\rho(T)$ enter this quantity which will henceforth be referred to as normalized imbibition speed $v_{\mathrm{n}}(T)$

$$
v_{\mathrm{n}}(T) \equiv \frac{C(T)}{C\left(T_{\mathrm{n}}\right)}=\frac{\rho(T)}{\rho\left(T_{\mathrm{n}}\right)} \cdot \sqrt{\frac{\sigma(T) \cdot \eta\left(T_{\mathrm{n}}\right)}{\sigma\left(T_{\mathrm{n}}\right) \cdot \eta(T)}} .
$$

One should not be confused by the term 'speed', since $v_{\mathrm{n}}$ is actually dimensionless. Nevertheless, it quantifies the mass uptake rate of the sample at a given temperature and a characteristic variation in any fluid property must be mapped in this quantity.

Here we present a thorough study on the capillary rise dynamics of the thermotropic liquid crystal octyloxycyanobiphenyl (8OCB) in mesopores. The building blocks of this liquid may be considered to be rigid rods with a length of $\sim 2 \mathrm{~nm}$ and diameter between $1 \mathrm{~nm}$ and $1.5 \mathrm{~nm}$. Bulk 8OCB undergoes a transition from the smectic A to the smectic $\mathrm{N}$ phase at $T=67^{\circ} \mathrm{C}$ and beyond the clearing point $T_{\mathrm{c}}=80^{\circ} \mathrm{C}$ any orientational ordering of the molecules disappears (isotropic phase). In particular this last transition 


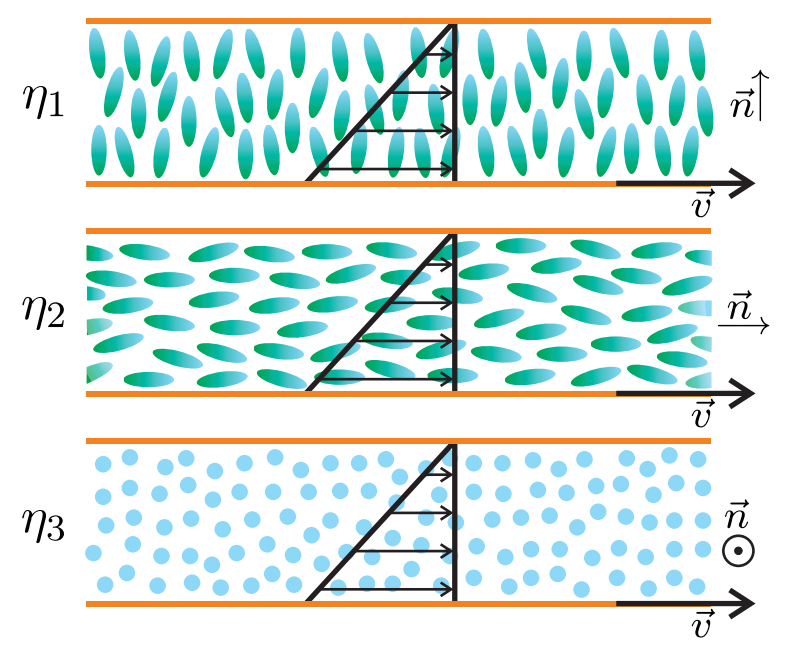

Figure 5: The experimental Couette flow conditions for measurements of the three Miesowicz shear viscosity coefficients of nematic liquid crystals: $\eta_{1}$ for $\vec{n} \perp \vec{v}$ and $\vec{n} \| \nabla v$, $\eta_{2}$ for $\vec{n} \| \vec{v}$ and $\vec{n} \perp \nabla v, \eta_{3}$ for $\vec{n} \perp \vec{v}$ and $\vec{n} \perp \nabla v$.

is accompanied by the occurrence of a characteristic shear viscosity minimum, what might uniquely influence the invasion dynamics. In the following this behavior will be described in detail.

\subsection{Nemato-Hydrodynamics: Shear viscosity minimum and presmectic divergence of flowing nematic liquid crystals}

The momentum transport in nematic liquid crystals shows an anisotropy since it depends on the mutual orientations of the macroscopic molecular alignment (the director $\vec{n}$ ), the flow velocity $(\vec{v})$ and the velocity gradient $(\nabla v)$. In 1946 Miesowicz defined three principal shear viscosity coefficients of nematics [70], which can be measured in three different Couette flow experiments sketched in Fig. 5. Typically magnetic fields are applied in order to align the molecules in the nematic sample. Intuition suggests that the lowest resistance to the nematic flow, i.e. the lowest viscosity value, should be $\eta_{2}$. Among the two remaining viscosities, $\eta_{1}$ should have the highest value.

Indeed, when the orienting magnetic field, i.e. the director $\vec{n}$, is parallel to the velocity $\vec{v}$ of the nematic flow, the lowest viscosity is recorded (see open symbols in Fig. 6). Nevertheless, this relatively simple picture of the viscosity of nematic liquid crystals is disturbed for the compounds exhibiting the transition to the smectic A phase. Then, with decreasing temperature, the viscosity $\eta_{2}$ shows a strong increase and goes to infinity at the N-A phase transition. The $\eta_{1}$ and $\eta_{3}$ viscosities are almost unaffected. At a temperature that is a few degrees below the temperature at which the transition to the smectic A phase takes place, the viscosities $\eta_{2}$ and $\eta_{3}$ interchange their roles and then the lowest nematic viscosity corresponds to the flow in the $\eta_{3}$ configuration. The presmectic behavior of the $\eta_{2}$ viscosity is due to the formation of precursors of smectic planes with $\vec{z} \| \vec{v}$ that would be immediately destroyed by the velocity gradient, thus 


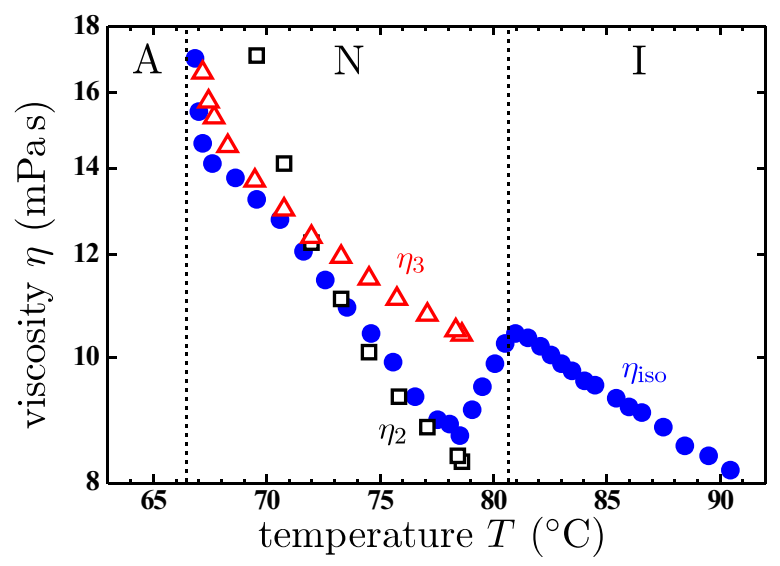

Figure 6: Miesowicz shear viscosities $\eta_{2}(\square)$ and $\eta_{3}(\triangle)$ of the liquid crystal 8 OCB compared to its free flow viscosity $\eta_{\text {iso }}(\bullet)$ according to $[71]$.

this configuration is rendered unfavorable.

The behavior of the freely flowing compound obeys a general principle that can be formulated as follows: a free fluid adopts such a manner of flow, as corresponds to the minimum of its viscosity at given conditions $[72$. Accordingly, the transition from the isotropic to the nematic phase manifests itself in a strong decrease of the shear viscosity $\eta_{\text {iso }}$ that is very close to $\eta_{2}$. Consistently, beyond the presmectic cross-over of $\eta_{2}$ and $\eta_{3}$ the viscosity of the freely flowing liquid crystal $\eta_{\text {iso }}$ follows $\eta_{3}$ (see filled symbols in Fig. 6). This result is interpreted in terms of rearrangements of the molecular alignment $\vec{n}$ with respect to the velocity field $\vec{v}$, which can easily be assessed by means of examinations of the compound's viscosity.

\subsection{Results and interpretation}

In Fig. 7 five measurements of $80 \mathrm{CB}$ invading V5 are shown along with $\sqrt{t}$-fits. Their coincidence is evident and hence the solution of the differential equation Eq. (8) through a $\sqrt{t}$-law is justified. This implicitly means that the right-hand side of Eq. (8) is not a function of the time at all, what has only tacidly been assumed so far. Especially the viscosity consequently is not a function of neither the time nor the time-dependent

shear rate, which is related to the rise speed $\frac{\mathrm{d} h(t)}{\mathrm{d} t} \propto \frac{1}{\sqrt{t}}$. Shear thinning or thickening effects of the liquid crystal can therefore be excluded.

The extracted normalized imbibition speeds $v_{\mathrm{n}}(T)$ are indicated by the single points in the upper panel of Fig. 8. Moreover, utilizing $\eta_{\text {iso }}(T)$-values according to Fig. 6 along with the known $T$-dependencies of $\sigma$ and $\rho$ one can also calculate $v_{\mathrm{n}}(T)$ (see solid line in the upper panel of Fig. 8).

The comparison between measurement and prediction reveals a variety of astonishing features. First of all, both V5 and V10 reveal comparable characteristics of the invasion dynamics of the liquid crystal 8OCB. Anyhow, only in the nematic phase the $T$-dependent behavior of the measured imbibition speeds coincides with the prediction 


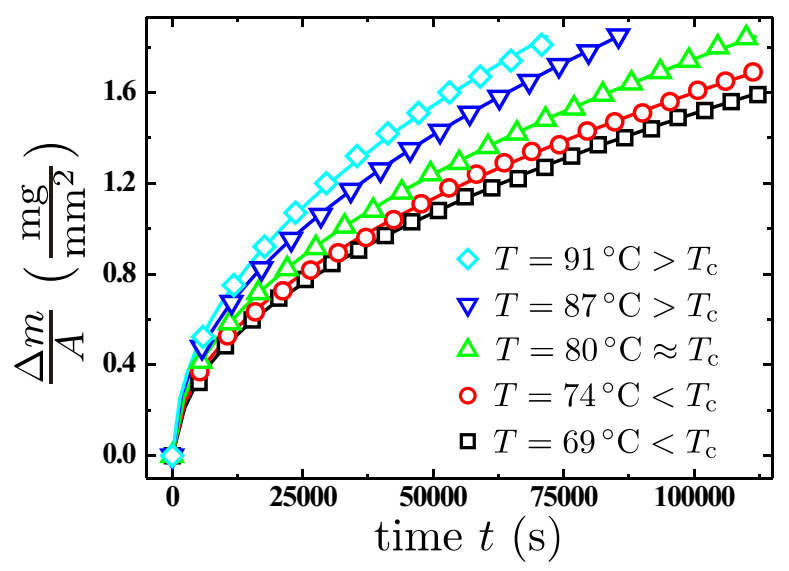

Figure 7: Specific mass uptake of V5 due to the imbibition of the liquid crystal 8OCB as a function of the time for selected temperatures below and above the clearing point $T_{\mathrm{c}} \approx 80^{\circ} \mathrm{C}$. Solid lines correspond to $\sqrt{t}$-fits. The data density is reduced by a factor of 2500 .

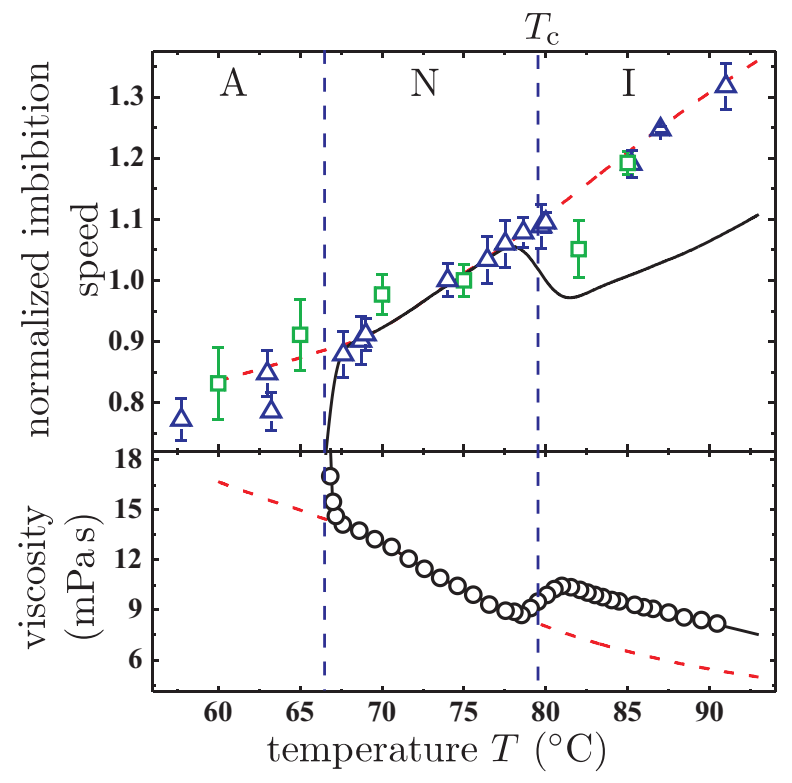

Figure 8: (upper panel): Measured normalized imbibition speeds $v_{\mathrm{n}}$ (for $T_{\mathrm{n}}=75^{\circ} \mathrm{C}$ ) of $80 \mathrm{CB}$ in V5 $(\triangle)$ and V10 $(\square)$, respectively, in comparison with values calculated on the basis of the viscosity values in the lower panel (-). (lower panel): $T$-dependent viscosity of $8 \mathrm{OCB}$ by way of comparison (see Fig. 6 for a detailed view). The dashed lines correspond to extrapolations of the calculated imbibition speeds and of the viscosity in the absence of the shear viscosity minimum and the presmectic divergence, respectively.

based on the $\eta_{\text {iso }}$ values presented in Fig. 6. In particular, the distinctive bump in the proximity of the clearing point $T_{\mathrm{c}}$ as the direct manifestation of the shear viscosity minimum in the theoretical behavior of $v_{\mathrm{n}}$ is unambiguously absent. The imbibition speed rather increases monotonously with increasing temperature. 
As mentioned before this distinctive feature of the viscosity at the clearing point is caused by the inset of an alignment of the molecules (in the nematic phase) with respect to the flow direction, that is $\vec{n} \| \vec{v}$. Therefore its absence can intuitively be interpreted in terms of an already existent alignment of the molecules beyond $T_{\mathrm{c}}$. It is obvious to conclude that such an alignment is easily induced by the extreme spatial confinement to cylindrical pores with diameters that are not more than five times the length of the molecule itself. In addition, considering their rigidity and, in particular, the parabolic flow profile established in the pore, it is hard to think of any alternative to the tendency of molecular alignment parallel to the pore axis and, consequently, to the flow velocity $\vec{v}$. From this point of view the absence of the shear viscosity minimum and, consistently, of the nematic to isotropic phase transition is not surprising at all but rather consequent. In this context it is more suitable to label the phase beyond the clearing point not isotropic but paranematic $(\mathrm{P})$. This term preeminently discloses the solely confinement-induced alignment of the liquid crystal.

It was demonstrated experimentally 7377 , in agreement with expectations from theory 78 80, that there is no 'true' I-N transition for liquid crystals confined in geometries spatially restricted in at least one direction to a few nanometers. The anchoring at the confining walls, quantified by a surface field, imposes a partially orientational, that is, a partially nematic alignment of the confined liquid crystal, even at temperatures $T$ far above the clearing point $T_{\mathrm{c}}$. The symmetry breaking does not occur spontaneously, as characteristic of a genuine phase transition, but is enforced over relevant distances by the interaction with the walls.

The assumption of such a paranemtic phase is corroborated by recent birefringence measurements of rod-like liquid crystals (7CB and $8 \mathrm{OCB}$ ) confined to an array of parallel, nontortuous channels of $10 \mathrm{~nm}$ mean diameter and $300 \mu \mathrm{m}$ length in a monolithic silica membrane 81,82 . These measurements elucidate that the surface anchoring fields render the bulk discontinuous I-N transition to a continuous P-N transition. The transition temperature $T_{\mathrm{c}}$ is found to be changed only marginally, due to a balance of its molecular alignment induced upward and its quenched disorder (attributable to wall irregularities) induced downward shift. This agrees with the observations of liquid crystals imbibed in tortuous pore networks [73, 74].

Interestingly, due to the complete absence of the shear viscosity minimum in the results of the $T$-dependent series of imbibition measurements shown in Fig. 8 a definition of a P-N transition temperature in confinement is not possible at all. This is confirmed by a simple extrapolation of the $\eta_{2}$ viscosity to higher $T$, which saliently reproduces the measured imbibition speeds beyond the bulk clearing point. This can only be interpreted in terms of an extremely high degree of orientation already existent in the paranematic phase; at least higher than suggested by the birefringence measurements [81]. This difference is most probably due to the basically differing detection method of the P-N transition: normally birefringence or calorimetry (DSC) measurements performed with the confined static liquid are applied for this purpose. But, the viscosity measurements presented here refer to the liquid's dynamics in mesopore confinement. 
As already mentioned before, the additional emerging flow velocity and in particular the velocity gradient seemingly enhance the paranematic orientational alignment in the $\eta_{2}$-configuration significantly.

The second remarkable feature of the results presented in Fig. 8 is the absence of the presmectic divergence of the viscosity of the freely flowing liquid crystal. This would result in a dramatic drop of the imbibition speed due to the inset of smectic layering. However, the measured values do not indicate such an effect. Its absence rather suggests a suppression of the A phase in favor of the $\mathrm{N}$ phase. This is elucidated by a simple extrapolation of the viscosity to lower $T$ as indicated in Fig. 8, which preeminently reproduces the measured imbibition speeds.

What are the reasons for this discovery? First of all, the confinement to a cylindrical pore (rather than to a film geometry like in the Couette flow depicted in Fig. 5) renders the $\eta_{3}$ viscosity as unfavorable as the $\eta_{1}$ viscosity. This again clarifies the high stability of the nematic $\eta_{2}$-configuration in confinement. A cross-over behavior as ascertained for the freely flowing bulk liquid can hence be excluded. Yet, the presmectic divergence of $\eta_{2}$ is caused by the destruction of precursors of smectic planes for $\vec{n} \| \vec{v}$. From this point of view the suppression of the A phase is not surprising at all but a mere consequence of the overall stabilization of the $\eta_{2}$-configuration in the mesopore confinement.

Even the static mesopore-confined liquid crystal shows such modified mesophase behavior [75]. For example the heat capacity anomaly typical of the second-order $\mathrm{N}$-A transition in rod-like liquid crystals immersed in aerogels is absent or greatly broadened [83,84]. Nuclear magnetic resonance (NMR) measurements revealed the lack of pretransitional smectic layering due to the rough surface of the confining walls [85]. Furthermore, a systematic study of the influence of the degree of confinement indicates that the $\mathrm{N}-\mathrm{A}$ transition becomes progressively suppressed with decreasing pore radius whereas the stability range of the nematic phase is increased [82, 86.

Finally we'd like to comment on results from an examination on the absolut invasion dynamics of the liquid crystal as opposed to the analysis of normalized imbibition coefficients just presented. This investigation was performed according to the procedure introduced in section 3.1 referring to mesopore-confined water. For the liquid crystal 8OCB we determined slip lengths of $b=(-1.11 \pm 0.23) \mathrm{nm}$ for V5 and $b=(-1.54 \pm 0.31) \mathrm{nm}$ for V10, respectively. Referring to the molecular shape stated earlier we may hence, again, conclude that one layer of flat lying molecules is pinned to the pore walls and does therefore not contribute to the flow dynamics. Apparent velocity slippage at the walls, as was expected to be associated with the molecular alignments in the channels [87], could not be detected in our study.

\section{Conclusions}

After a short introduction of liquid imbibition in porous hosts we have presented a gravimetric study on spontaneous imbibition of water and the liquid crystal 8OCB. We inferred from our studies an interfacial boundary layer adjacent to the pore walls with 
a defined thickness whose dynamics are mainly determined by the interaction between liquid and substrate. This manifests itself in terms of a negative velocity slip length for both liquids investigated. The flow properties of the pore-condensed molecules in the pore center are, however, remarkable robust, that is bulk-like or at least similar to the bulk phase (in the case of the liquid crystal).

The $T$-dependent imbibition measurements of the liquid crystal $80 \mathrm{CB}$ revealed that confinement plays a similar role as an external magnetic field for a spin system: the strong first-order I-N transition is replaced by a weak first-order or continuous paranematic to nematic transition, depending on the strength of the surface orientational field [88]. Based on detailed knowledge of the static (equilibrated) liquid's behavior in the mesopores as deduced from previously conducted birefringence experiments, we were able to procure complementary results with respect to its dynamic (non-equilibrium) behavior. The additional emerging flow velocity and in particular the velocity gradient enhance the paranematic orientational alignment significantly rendering the P-N transition even broader than known from the equilibrium state. Due to the high stabilization of the $\eta_{2}$-configuration in the $\mathrm{N}$ phase the $\mathrm{A}$ phase is suppressed and the stability range of the nematic phase is increased.

The finding of two distinct species (with regard to flow dynamics) is reminiscent of the partitioning of pore condensates found in vapour sorption isotherms (filmcondensed versus capillary-condensed state) [89], of the two species often reported in studies on self-diffusions dynamics (slowed-down or increased self-diffusion versus bulk dynamics) 69,90,92], of the effects of pore condensates on the deformation of the matrix (expansion versus contraction of the pore walls upon change from filmcondensed to capillary-condensed state) [93]. Last but not least, it reminds of the distinct crystallization behaviour of pore condensates (an amorphous boundary layer versus a crystallized fraction of molecules in the pore center) [89]. In principle, this partitioning, but also the highly stabilized nematic phase of a confined liquid crystal, can be traced to the strong influence of the substrate potential on the liquid or solid layers, respectively, right adjacent to the pore walls 94 96.

From a more general perspective, our experimental studies demonstrate that an understanding of the non-equilibrium behavior of confined soft-matter system necessitates a thorough understanding of their equilibrium behavior, which quite often differs markedly from the macroscopic phenomenology 97,98 .

\section{Acknowledgments}

It is a great pleasure to thank Andriy Kityk, Klaus Knorr, Dirk Wallacher, and Howard A. Stone for stimulating discussions. Financial support by the DFG under grants Hu850/2 (1-2) within the priority program 'Nano- and Microfluidics' is acknowledged. 


\section{References}

[1] Bear J. Dynamics of Fluids in Porous Media (Dover, New York (USA), 1988).

[2] Koch GW, Sillett SC, Jennings GM, and Davis SD. The limits to tree height. Nature 428, 851 (2004).

[3] Wheeler TD and Stroock AD. The transpiration of water at negative pressures in a synthetic tree. Nature 455, 208 (2008).

[4] Sahimi M. Flow phenomena in rocks - From continuum models to fractals, percolation, cellularautomata, and simulated annealing. Rev. Mod. Phys. 65 (4), 1393 (1993).

[5] Mecke K and Arns CH. Fluids in porous media: A morphometric approach. J. Phys.: Condens. Matter 17 (9), S503 (2005).

[6] Alava M, Dube M, and Rost M. Imbibition in disordered media. Adv. Phys. 53, 83 (2004).

[7] Sparreboom W, van den Berg A, and Eijkel JCT. Principles and applications of nanofluidic transport. Nat. Nanotechnol. 4, 713 (2009).

[8] Eijkel JCT and van den Berg A. Nanofluidics: what is it and what can we expect from it? Microfluid. Nanofluid. 1, 249 (2005).

[9] Salieb-Beugelaar GB, Simone G, Arora A, Philippi A, and Manz A. Latest developments in microfluidic cell biology and analysis systems. Anal. Chem. (in press) (2010).

[10] Arora A, Simone G, Salieb-Beugelaar GB, Kim JT, and Manz A. Latest developments in micro total analysis systems. Anal. Chem. (in press) (2010).

[11] Thomas A, Goettmann F, and Antonietti M. Hard templates for soft materials: Creating nanostructured organic materials 20, 738 (2008).

[12] Luo Y, Lee SK, Hofmeister H, Steinhart M, and Gösele U. Pt nanoshell tubes by template wetting. Nano Lett. 4, 143 (2004).

[13] Elmer TH. Porous and reconstructed glasses, volume 4 of Engineered Materials Handbook, page 427 (ASM International, Materials Park, OH, 1992).

[14] Gruener S. Rheology and Dynamics of Simple and Complex Liquids in Mesoporous Matrices. Ph.D. thesis, Saarland University, Saarbrücken, Germany (2010).

[15] Saam WF and Cole MW. Excitations and thermodynamics for liquid-helium films. Phys. Rev. B 11, 1086 (1975).

[16] Lin MY, Abeles B, Huang JS, Stasiewski HE, and Zhang Q. Visocus flow and diffusion of liquids in microporous glasses. Phys. Rev. B 46, 10701 (1992).

[17] Bommer S. Gas- und Proteinpermeabilitätsmessungen an biomimetischen Festkörpermembranen. Diploma thesis, Saarland University, Saarbrücken, Germany (2008).

[18] Crossley RA, Schwartz LM, and Banavar JR. Image-based models of porous media: Application to Vycor glass and carbonate rocks. Appl. Phys. Lett. 59, 3553 (1991).

[19] Debye P and Cleland RL. Flow of liquid hydrocarbons in porous Vycor. J. Appl. Phys. 30 (6), 843 (1959).

[20] Granick S, Zhu YX, and Lee H. Slippery questions about complex fluids flowing past solids. Nat. Mater. 2, 221 (2003).

[21] Lauga E, Brenner MP, and Stone HA. Handbook of Experimental Fluid Dynamics, chapter 15 Microfluidics: The No-Slip Boundary Condition (Springer, New York, 2006).

[22] Neto C, Evans DR, Bonaccurso E, Butt HJ, and Craig VSJ. Boundary slip in Newtonian liquids: A review of experimental studies. Rep. Prog. Phys. 68, 2859 (2005).

[23] Barrat JL and Bocquet L. Large slip effect at a nonwetting fluid-solid interface. Phys. Rev. Lett. 82, 4671 (1999).

[24] Pit R, Hervet H, and Leger L. Direct experimental evidence of slip in hexadecane: Solid interfaces. Phys. Rev. Lett. 85, 980 (2000).

[25] Cieplak M, Koplik J, and Banavar JR. Boundary conditions at a fluid-solid interface. Phys. Rev. Lett. 86, 803 (2001).

[26] Tretheway DC and Meinhart CD. Apparent fluid slip at hydrophobic microchannel walls. Phys. 
Fluids 14, L9 (2002).

[27] Cho JHJ, Law BM, and Rieutord F. Dipole-dependent slip of Newtonian liquids at smooth solid hydrophobic surfaces. Phys. Rev. Lett. 92, 166102 (2004).

[28] Schmatko T, Hervet H, and Leger L. Friction and slip at simple fluid-solid interfaces: The roles of the molecular shape and the solid-liquid interaction. Phys. Rev. Lett. 94, 244501 (2005).

[29] Fetzer R and Jacobs K. Slippage of Newtonian liquids: Influence on the dynamics of dewetting thin films. Langmuir 23, 11617 (2007).

[30] Bäumchen $\mathrm{O}$ and Jacobs K. Slip effects in polymer thin films. J. Phys.: Condens. Matter 22, $033102(2010)$.

[31] Voronov RS, Papavassiliou DV, and Lee LL. Review of fluid slip over superhydrophobic surfaces and its dependence on the contact angle. Ind. Eng. Chem. Res. 47, 2455 (2008).

[32] Maali A, Cohen-Bouhacina T, and Kellay H. Measurement of the slip length of water flow on graphite surface. Appl. Phys. Lett. 92, 053101 (2008).

[33] Servantie J and Müller M. Temperature dependence of the slip length in polymer melts at attractive surfaces. Phys. Rev. Lett. 101, 026101 (2008).

[34] Sendner C, Horinek D, Bocquet L, and Netz RR. Interfacial water at hydrophobic and hydrophilic surfaces: Slip, viscosity, and diffusion. Langmuir 25, 10768 (2009).

[35] Zhu Y and Granick S. Rate-dependent slip of Newtonian liquid at smooth surfaces. Phys. Rev. Lett. 87, 096105 (2001).

[36] Craig VSJ, Neto C, and Williams DRM. Shear-dependent boundary slip in an aqueous Newtonian liquid. Phys. Rev. Lett. 87, 054504 (2001).

[37] Priezjev NV and Troian SM. Molecular origin and dynamic behavior of slip in sheared polymer films. Phys. Rev. Lett. 92, 018302 (2004).

[38] Priezjev NV. Rate-dependent slip boundary conditions for simple fluids. Phys. Rev. E 75, 051605 (2007).

[39] Vinogradova OI and Yakubov GE. Surface roughness and hydrodynamic boundary conditions. Phys. Rev. E 73, 045302(R) (2006).

[40] Zhu Y and Granick S. Limits of the hydrodynamic no-slip boundary condition. Phys. Rev. Lett. 88, 106102 (2002).

[41] Bonaccurso E, Butt HJ, and Craig VSJ. Surface roughness and hydrodynamic boundary slip of a Newtonian fluid in a completely wetting system. Phys. Rev. Lett. 90, 144501 (2003).

[42] Dammer SM and Lohse D. Gas enrichment at liquid-wall interfaces. Phys. Rev. Lett. 96, 206101 (2006).

[43] Cheikh C and Koper G. Stick-slip transition at the nanometer scale. Phys. Rev. Lett. 91, 156102 (2003).

[44] Caupin F, Cole MW, Balibar S, and Treiner J. Absolute limit for the capillary rise of a fluid. Europhys. Lett. 82, 56004 (2008).

[45] Lucas R. Über das Zeitgesetz des kapillaren Aufstiegs von Flüssigkeiten. Kolloid Zeitschrift 23, 15 (1918).

[46] Washburn EW. The dynamics of capillary flow. Phys. Rev. 17, 273 (1921).

[47] Gelb LD and Hopkins AC. Dynamics of the capillary rise in nanocylinders. Nano Lett. 2 (11), 1281 (2002).

[48] Dimitrov DI, Milchev A, and Binder K. Capillary rise in nanopores: Molecular dynamics evidence for the Lucas-Washburn equation. Phys. Rev. Lett. 99, 054501 (2007).

[49] Israelachvili J. Measurement of the viscosity of liquids in very thin films. J. Colloid Interface Sci. 110, 263 (1986).

[50] Horn RG, Smith DT, and Haller W. Surface forces and viscosity of water measured between silica sheets. Chem. Phys. Lett. 162, 404 (1989).

[51] Raviv U, Laurat P, and Klein J. Fluidity of water confined to subnanometre films. Nature 413 (6851), 51 (2001).

[52] Fisher LR, Gamble RA, and Middlehurst J. The Kelvin equation and the capillary condensation 
of water. Nature 290, 575 (1981).

[53] Fradin $\mathrm{C}$ et al. Reduction in the surface energy of liquid interfaces at short length scales. Nature 403, 871 (2000).

[54] Heinbuch U and Fischer J. Liquid flow in pores: Slip, no-slip, or multilayer sticking. Phys. Rev. A 40, 1144 (1989).

[55] Ricci MA, Bruni F, Gallo P, Rovere M, and Soper AK. Water in confined geometries: Experiments and simulations. J. Phys.: Condens. Matter 12, A345 (2000).

[56] Gallo P, Rovere M, and Spohr E. Supercooled confined water and the mode coupling crossover temperature. Phys. Rev. Lett. 85, 4317 (2000).

[57] Vichit-Vadakan W and Scherer GW. Measuring permeability of rigid materials by a beam-bending method: II, Porous glass. J. Am. Ceram. Soc. 83, 2240 (2000).

[58] Gallo P, Ricci MA, and Rovere M. Layer analysis of the structure of water confined in Vycor glass. J. Chem. Phys. 116, 342 (2002).

[59] Castrillon SRV, Giovambattista N, Aksay IA, and Debenedetti PG. Effect of surface polarity on the structure and dynamics of water in nanoscale confinement. J. Phys. Chem. B 113, 1438 (2009).

[60] Fouzri A, Dorbez-Sridi R, and Oumezzine M. Water confined in silica gel and in Vycor glass at low and room temperature, X-ray diffraction study. J. Chem. Phys. 116, 791 (2002).

[61] Li TD, Gao J, Szoszkiewicz R, Landman U, and Riedo E. Structured and viscous water in subnanometer gaps. Phys. Rev. B 75, 115415 (2007).

[62] Khan SH, Matei G, Patil S, and Hoffmann PM. Dynamic solidification in nanoconfined water films. Phys. Rev. Lett. 105, 106101 (2010).

[63] Xu K, Cao P, and Heath JR. Graphene visualizes the first water adlayers on mica at ambient conditions. Science 329, 1188 (2010).

[64] Lasne D et al. Velocity profiles of water flowing past solid glass surfaces using fluorescent nanoparticles and molecules as velocity probes. Phys. Rev. Lett. 100, 214502 (2008).

[65] Vinogradova OI. Slippage of water over hydrophobic surfaces. Int. J. Miner. Process. 56, 31 (1999).

[66] Gruener S, Hofmann T, Wallacher D, Kityk AV, and Huber P. Capillary rise of water in hydrophilic nanopores. Phys. Rev. E 79, 067301 (2009).

[67] Huber P, Gruener S, Schaefer C, Knorr K, and Kityk AV. Rheology of liquids in nanopores: A study on the capillary rise of water, n-hexadecane and n-tetracosane in mesoporous silica. Eur. Phys. J. Special Topics 141, 101 (2007).

[68] Gruener S and Huber P. Spontaneous imbibition dynamics of an n-alkane in nanopores: Evidence of meniscus freezing and monolayer sticking. Phys. Rev. Lett. 103, 174501 (2009).

[69] Kusmin A et al. Evidence of a sticky boundary layer in nanochannels: A neutron spin echo study of n-hexatriacontane and poly(ethylene oxide) confined in porous silicon. J. Phys. Chem. Lett. 1, $3116(2010)$.

[70] Miesowicz M. The 3 coefficients of viscosity of anisotropic liquids. Nature 158, 261 (1946).

[71] Graf HH, Kneppe H, and Schneider F. Shear and rotational viscosity coefficients of two nematic liquid crystals. Mol. Phys. 77, 521 (1992).

[72] Jadzyn J and Czechowski G. The shear viscosity minimum of freely flowing nematic liquid crystals. J. Phys.: Condens. Matter 13, L261 (2001).

[73] Iannacchione GS, Crawford GP, Zumer S, Doane JW, and Finotello D. Randomly constrained orientational order in porous glass. Phys. Rev. Lett. 71, 2595 (1993).

[74] Dadmun MD and Muthukumar M. The nematic to isotropic transition of a liquid crystal in porous media. J. Chem. Phys. 98, 4850 (1993).

[75] Iannacchione GS and Finotello D. Specific heat dependence on orientational order at cylindrically confined liquid crystal phase transitions. Phys. Rev. E 50, 4780 (1994).

[76] Crandall KA, Rosenblatt C, and Aliev FM. Ellipsometry at the nematic-isotropic phase transition in a confined geometry. Phys. Rev. E 53, 636 (1996). 
[77] Cloutier SG et al. Molecular self-organization in cylindrical nanocavities. Phys. Rev. E 73, 051703 (2006).

[78] Sheng P. Phase transition in surface-aligned nematic films. Phys. Rev. Lett. 37, 1059 (1976).

[79] Steuer H, Hess S, and Schoen M. Phase behavior of liquid crystals confined by smooth walls. Phys. Rev. E 69, 031708 (2004).

[80] Cheung DL and Schmid F. Isotropic-nematic transition in liquid crystals confined between rough walls. Chem. Phys. Lett. 418, 392 (2006).

[81] Kityk AV et al. Continuous paranematic-to-nematic ordering transitions of liquid crystals in tubular silica nanochannels. Phys. Rev. Lett. 101, 187801 (2008).

[82] Kityk AV and Huber P. Thermotropic nematic and smectic order in silica glass nanochannels. Appl. Phys. Lett. 97, 153124 (2010).

[83] Bellini T, Radzihovsky L, Toner J, and Clark NA. Universality and scaling in the disordering of a smectic liquid crystal. Science 294, 1074 (2001).

[84] Qian S, Iannacchione GS, and Finotello D. Critical behavior of a smectic-A to nematic phase transition imbedded in a random network of voids. Phys. Rev. E 57, 4305 (1998).

[85] Ondris-Crawford RJ, Crawford GP, Doane JW, and Zumer S. Surface molecular anchoring in microconfined liquid crystals near the nematic-smectic-A transition. Phys. Rev. E 48, 1998 (1993).

[86] Kutnjak Z, Kralj S, Lahajnar G, and Zumer S. Calorimetric study of octylcyanobiphenyl liquid crystal confined to a controlled-pore glass. Phys. Rev. E 68, 021705 (2003).

[87] Heidenreich S, Ilg P, and Hess S. Boundary conditions for fluids with internal orientational degrees of freedom: Apparent velocity slip associated with the molecular alignment. Phys. Rev. E 75, $066302(2007)$.

[88] Stark H. Saturn-ring defects around microspheres suspended in nematic liquid crystals: An analogy between confined geometries and magnetic fields. Phys. Rev. E 66, 032701 (2002).

[89] Huber P and Knorr K. Adsorption-desorption isotherms and x-ray diffraction of Ar condensed into a porous glass matrix. Phys. Rev. B 60, 12657 (1999).

[90] Baumert J, Asmussen B, Gutt C, and Kahn R. Pore-size dependence of the self-diffusion of hexane in silica gels. J. Chem. Phys. 116 (24), 10869 (2002).

[91] Koppensteiner J, Schranz W, and Puica MR. Confinement effects on glass forming liquids probed by dynamic mechanical analysis. Phys. Rev. B 78, 054203 (2008).

[92] Kusmin A et al. Polymer dynamics in nanochannels of porous silicon: A neutron spin echo study. Macromolecules 43, 8162 (2010).

[93] Guenther G, Prass J, Paris O, and Schoen M. Novel insights into nanopore deformation caused by capillary condensation. Phys. Rev. Lett. 101 (8), 086104 (2008).

[94] Scheidler P, Kob W, and Binder K. The relaxation dynamics of a simple glass former confined in a pore. Europhys. Lett. 52, 277 (2000).

[95] Scheidler P, Kob W, and Binder K. Cooperative motion and growing length scales in supercooled confined liquids. Europhys. Lett. 59, 701 (2002).

[96] Klapp SL and Schoen M. Spontaneous orientational order in confined dipolar fluid films. J. Chem. Phys. 117, 8050 (2002).

[97] Binder K, Horbach J, Vink R, and Virgiliis AD. Confinement effects on phase behavior of soft matter systems. Soft Matter 4, 1555 (2008).

[98] Shen AQ and Cheung P. The freedom of confinement in complex fluids. Phys. Today 63, 30 (2010). 